Johannes G. Schmidt

Auch vegetarische Ernährung ist Gift oder Heilmittel - es kommt darauf an, für wen und wann. Die angebliche wissenschaftliche Evidenz, auf die sich der vegetarische Ärztekongress beruft, ist anfällig für Trugschlüsse. Kohortenstudien unterliegen immer einem möglichen "Confounding Bias», und so sagt die unbestrittene Tatsache, dass Vegetarier länger leben, weniger übergewichtig sind und weniger Diabetes aufweisen noch nicht, ob diese gesundheitlichen Vorteile als Folge der vegetarischen Ernährung bestehen oder trotz der vegetarischen Ernährung. Vielleicht würden Vegetarier noch länger leben, wenn sie etwas Fleisch ässen.

Nach der altchinesischen Medizin und Ernährungslehre gibt es Menschen, die kein oder kaum Fleisch benötigen, weil sie von Geburt an viel Yang - Lebensfeuer - haben. Und diese Menschen mit ihrem angeborenen starken Lebensfeuer sind widerstandsfähig und werden alt, ob sie nun vegetarisch leben oder nicht; sie können gut auf Fleisch verzichten. Fleisch macht stark und manchmal hitzig. Wenn daraus Aggressivität und Ungeduld resultiert, ist ein Verzicht auf Fleisch günstig; wenn die Stärkung sich produktiv auswirkt, ist Fleisch wieder nützlich. Hingegen ist für Menschen mit wenig Yang oder mit erschöpftem Yang eine Ernährung mit Fleisch die beste Medizin, das mangelnde Yang aufzubauen und möglichst starkzuhalten.

Vegetarianismus ist derzeit eine Mode in einer urban unruhigen Welt, die ihre Mitte verloren hat und das

\title{
Zu Gehrke M: Årztekongress VegMed 2012: Vegetarische Ernährung und Medizin - solide Evidenz, aber bessere Umsetzung in die Praxis nötig. Schweiz Z Ganzheitsmed 2013;25:220-222
}

Heil sucht. Vegetarianismus ist in keiner Weise Naturheilkunde. Medizinische Moden folgen immer der gleichen Überinterpretation von ungeeigneten Studien. Vegetarier sind wie gesagt eine Selektion von Menschen, sodass die scheinbaren gesundheitlichen Vorteile das Ergebnis einer statistischen Täuschung (Confounding Bias) sein können. Zudem gibt es auch Vegetarier mit Übergewicht und Diabetes sowie viele Fleischesser ohne Übergewicht und Diabetes. Die unkritische Verallgemeinerung von (scheinbaren) Risikofaktoren auf den Patienten-Einzelfall ist ein Irrtum, der nun nicht auch noch die Naturheilkunde besetzen muss. Weil wir in der altchinesischen Medizin und in der Naturheilkunde bei guter diagnostischer und praktischer Erfahrung wissen, dass die Ernährung auf den $\mathrm{Pa}$ tienten-Einzelfall abgestimmt sein muss, sehen wir, dass statistische Durchschnitte uns klinisch irreführen. Wir sehen viele übergewichtige Patienten mit Milz-Yang-Schwäche, die erst mit einer Yang-aufbauenden Ernährung, zu der etwa Fleischbrühen (Kraftbrühen) gehören, eine nachhaltige Überwindung der müden Verdauung und die Elimination von Kälte und Nässe erreichen. Das dauert oft 3-4 Jahre und länger. Das Kochen ist überdies genauso wichtig wie die gewählten Nahrungsmittel. Bei Milz-
Yang-Schwäche haben Vegetarier einen grossen Nachteil, der sich durch gutes Kochen und Yang-aufbauende Kräuter nur einigermassen ausgleichen lässt.

Erschöpfte Menschen brauchen mehr Fleisch, und die meisten Menschen leiden bei der heutigen Lebensweise an irgendeiner Form von Yang-Erschöpfung. Dass wir viel Fleisch essen, um eine hektische, egoistische und kräftezehrende Lebensweise durchhalten zu können, ist nicht unbedingt ein glücklicher Umstand. Ein Mensch wird umsichtiger und zufriedener, wenn er gut regeneriert ist und seine Kräfte bewahrt. Deshalb und aus Rücksicht auf die Umwelt ist es sicherlich vorteilhaft, dass wir mit weniger Fleischverzehr leben lernen. Auf dem Weg dahin müssen wir aber in den meisten Fällen (in einer Art «Erstverschlimmerung») genug Fleisch essen, um unsere YangErschöpfung in Ordnung zu bringen.

Die vorhandenen Studien zeigen nicht, dass eine vegetarische Ernährung die Ursache für eine höhere Lebenserwartung ist. Sie lassen vielmehr auch die Möglichkeit zu, dass eine starke Konstitution einerseits Fleischverzicht begünstigt und andererseits per se vor Krankheiten schützt. Vermutlich wählen heute oft die falschen Personen eine vegetarische Ernährung.

\section{KARGER}

Fax +49761 4520714
(๑) 2013 S. Karger GmbH, Freiburg

Accessible online at: www.karger.com/szg
Dr. med. Johannes G. Schmidt

Stiftung Paracelsus heute

Praxiszentrum Meinradsberg

Ilgenweidstrasse 3, 8840 Einsiedeln, Schweiz

schmidt@paracelsus-heute.ch 